\title{
HoloGCS : Mixed Reality-based Ground Control Station for Unmanned Aerial Vehicle
}

This paper was downloaded from TechRxiv (https://www.techrxiv.org).

\section{LICENSE}

CC BY 4.0

SUBMISSION DATE / POSTED DATE

05-01-2022 / 07-01-2022

\section{CITATION}

Widiyanti, Daniar Estu; Asmoro, Krisma; Shin, Soo Young (2022): HoloGCS : Mixed Reality-based Ground Control Station for Unmanned Aerial Vehicle. TechRxiv. Preprint.

https://doi.org/10.36227/techrxiv.17869082.v1

DOI

10.36227/techrxiv.17869082.v1 


\title{
HoloGCS : Mixed Reality-based Ground Control Station for Unmanned Aerial Vehicle
}

\author{
Daniar Estu Widiyanti, Krisma Asmoro and Soo Young Shin, Senior Member, IEEE
}

\begin{abstract}
Ground control station (GCS) is a system for controlling and monitoring unmanned aerial vehicle (UAV). In current GCS, the device used are considered as complex environment. This paper proposes a video streaming and speech command control for supporting mixed reality based UAV GCS using Microsoft HoloLens. Video streaming will inform the UAV view and transmit the raw video to the HoloLens, while the HoloLens steers the UAV based on the displayed UAV field of view (FoV). Using the HoloLens Mixed Reality Tool-Kit (MRTK) speech input, UAV speech control from the HoloLens was successfully implemented. Finally, experimental results based on video streaming and speech command calculation of the throughput, round-time trip, latency and speech accuracy tests are discussed to demonstrate the feasibility of the proposed scheme.
\end{abstract}

Index Terms-Mixed Reality, Unmanned Aerial Vehicle, Ground Control Station, Speech Control, Video Streaming, Microsoft HoloLens.

\section{INTRODUCTION}

$\mathrm{T}$ HE 4.0 industrial revolution has introduced a paradigm shift towards smart factory that utilizes a new approach to achieve mass production, supported by low-cost effective product devices that lead the conventional factory to evolve [1]. Smart factories depend on the auto-decision making that is performed by all interconnected devices with the aid of artificial intelligence (AI) [2] and robot implementation [3] to enhance the production rate even more. On the other hand, human roles in smart factories were mainly focused on monitoring and maintaining devices.

In a smart factory, several machines and devices are connected to communicate; therefore, it will be challenging for a human or operator to maintain all of it. Recently, augmented reality (AR) and mixed reality (MR) as headmounted displays (HMDs) with intuitive interactions are the most likely strong candidate devices in the future to aid humans in smart factories in order to control robots [4], [5]. Several portable devices that exploit the MR principle have been developed to enhance the ease of human-machine interaction (HMI), two of which are conventional smart glasses [6] and Microsoft HoloLens as one of the most powerful smart glasses. HoloLens has numerous advantages compare to smart glass. It is provide a broader field of view (FoV), better battery lifespan and a more ergonomic head-fit. Moreover, with the faster clock speed of the central processing unit (CPU), Hololens capable to recognize and process human interaction inputs (gaze, gesture, voice) faster [7]. The implementation of HoloLens to assist HMI has been done before, such as gesture tracking with the precise and fast fingertip tracking to get a 2 dimension position $(x, y$ coordinates) of a mobile robot and mapping it to a $3 \mathrm{D}$ space

- D. E. Widiyanti, K. Asmoro and S. Y. Shin are with the Wireless and Emerging Network Systems Laboratory, Department of IT Convergence Engineering, Kumoh National Institute of Technology, Gumi 39177, South Korea (e-mail: daniarestuw@kumoh.ac.kr, krisma@kumoh.ac.kr, wdragon@kumoh.ac.kr). to mark the location of the robot $[8]$.

The other implementation of HMI is to control the movement of various robots in smart factories has been done, especially by controlling a mobile robot. In [9], a UAV as a mobile robot has gained huge popularity owing to its capabilities to hover and allow 3-dimensional movement. The UAV appeared on Industry 4.0, as an intelligent device that has changed the rules of the game in the industrial sector, providing benefits through innovative applications. UAVs have been utilized for monitoring in many sectors, such as, construction [10], mining [11] and city roads [12].

Although UAVs can work autonomously in a smart factory, an operator must monitor and observe to take over the control if it is misbehaving. To take control of the UAV movement, currently, a conventional wireless remote controller (RC) is most likely used by the operator. However, this is causing a problem since its difficult to use for firsttime users and can only be manipulated by experts [13]. As a result, a diverse approach regarding study to replace conventional wireless RC for UAV maneuver have been tested and implemented. Moreover, the human movements (gestures), voices and brain waves based wireless RC are developed to overcome the problem [14|. The voice-based control system has been proposed by [15], the work presented an AI based voice recognition to control a UAV. Another mobile robot control can be seen in [16], the system proposed a remote control for a mobile robot based on android application which integrated with web-page. The web-page include video screen for surveillance and buttons to control the mobile robot and the camera. The robot can move around and the operator can see a MJPG streamer program through a HTTP session live from the robot.

In order to precisely control the movement of the UAV by an operator with an HMD, a video streaming system is required. Previously, HoloLens video streaming from camera that mounted on a UAV was performed in [17] and [18]. Video streaming is important because the steering is based on the decision-making from live video streaming that the 
user can see in the application. Based on this purposes, a video streaming system is added to the system to allow the HMD user to control the UAV based on what the user sees easily. In this regard, the main purpose of this study is to propose an MR-based UAV ground control station. For this purpose, the system details and implementation are thoroughly explained. The main contributions of this work can be summarized as follows:

- A Video streaming system which forward the UAV view to be seen in live on the HMD

- A Speech command system in the HMD that could control the UAV

- Performance evaluation of throughput, round-trip time, and latency for the proposed system

- Performance evaluation of speech accuracy to test the reliability of the proposed system

The remainder of this paper is structured as follows. Section II examines previous research on current remote controller and video streaming methods. Section III focuses on the details of the proposed system. Section IV explains the implementation process in detail. Section $\mathrm{V}$ discusses the details of the measured throughput, round-trip time, and latency. Finally, section VI elucidates the entire article.

\section{Related Work}

Currently controlling a UAV as mobile robot can be performed in multiple ways. Some previous studies used a different type of controller to control the robots. In [19], remote control for mobile robots based on wireless transmission was proposed. The wireless transmission was used for data transfer. The system consists of a control center, a mobile robot and a display terminal. The control system is videobased control, wherein the live video is transmitted by the robot and is used by the operator to control the robot.

Studies in [20] proposed a AVR-based mobile robot remote controller using low cost remote controller Integrated Circuits (IC) in surveillance case. The system includes obstacle avoidance using ultrasonic distance sensor. The system also includes a live video and image, where the robot will move around and the operator can see a live video from the robot. Raspberry pi are used for developing the system.

Another study can be found in |21|. They proposed a robot remote control system using an Android-based mobile device. The remote control device was a mobile phone with a trackball concept. The user can control the remote by using a touch display or voice. Voice control can be used only when the user is connected to the Internet. The user can monitor the distance between the robot and the obstacle while displaying the view on the mobile device.

Focusing on voice control, [22] proposed voice control using an artificial neural network (ANN)-based automatic speech recognition (ASR) engine. They proposed a forwardfeed neural network-based speech recognition method for voice-controlled micro air vehicle (MAV) applications. Their system is a machine learning-based system in which the speech must be pre-trained before it can be used. Their system further developed a connected ASR word that has been trained to generate a command for controlling the MAV.
Another voice control robot is proposed by |23|. They proposed an autonomous voice control for robot which the robot can instantly move automatically without hitting any obstacle using the ultrasonic sensors. Their robot could help the users to give a general views of the robot without have to worry about any obstacle. They claims the robot system could be adaptable for people with wheelchair to make an autonomous wheelchair.

A car-based speech recognition control system was proposed in [24]. They used multiple chip modules and developed system to make an intelligent speech control system. They also added ultrasonic obstacle avoidance to their system. They combined their speech control system and obstacle avoidance module to control car movement. The average speech completion of their system was $46,67 \%$. With voice control, the car could avoid any obstacles encountered.

In [25], voice control in ground control station (GCS) of an UAV to solve the increasing complexity of UAV missions. Speech recognition processing software and seat voice control software was used on the system. The voice control systems use software that classifies self-inspection, speech recognition, Ethernet communication, speech model loading, online upgrade and automatic testing.

Some studies have reported the use of voice control to control their mobile robots. Expecting the use of voices for controller claims to minimize a key that was incorrectly entered. In this case, voice activation can help avoid these issues. Most previous studies proposed transferring interaction from natural language to robotic movement. This means that communication between humans and robots humans can easily adapt and understand by the robot.

Another study proposed a natural language-based control for UAVs [26]. The system combines a-natural language processing inside an MR environment to solve tasks and process navigational instructions given to the autonomous UAV. This work allows the UAV to be controlled with both voices and gestures. The use of voice control is limited, and only available when the user is connected to the Internet. The work claims to observe the UAVs movement and the situation in the real world while performing the task as per the issued commands.

As previously mentioned in [19], a live video is required to control the system because the steering is based on the video. In this case, a video streaming system is also required to develop an efficient and easy control method. Some video streaming works have multiple ways to forward views from one device to another device.

Previous work [27] also recommends the use of a smart helmet that responds to accidents in order to rescue personnel in the case of a disaster. The smart helmets core system equipment, collects, produces, and interprets data from attached sensors such as an infrared camera, residual oxygen sensors and a UAV camera to determine the rescue scenario, which can be observed via HMD and the command center.

Another study can be found in |28|. They proposed a Multi-RAT approach for maximizing communication reliability for Search and Rescue robots in challenging environment. The system consist of $8 \mathrm{~K}$ surround cameras and, a Wi-Fi LTE modems. Their system claims the robot can successfully tele-operated and streamed a high quality image data of the environment. 


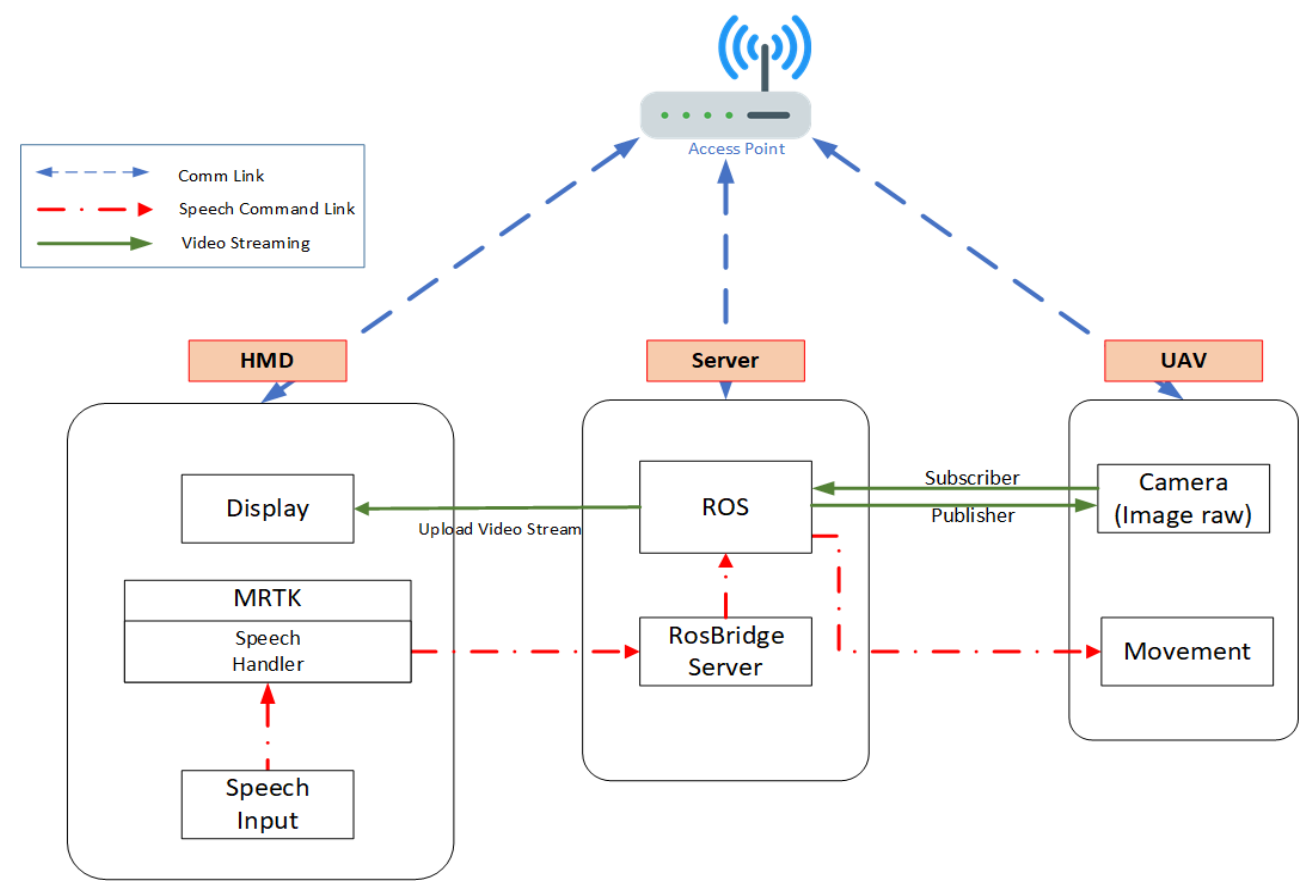

Fig. 1. The overall system model of this paper. UAV, Server and Head-Mounted Device (HMD) are connected to same Access Point

Other previous studies also can be seen in [29]. They proposed an Electroencephalography (EEG)-based brain computer interaction control system. They use a beta waveforms of the user to guides the motion of a robot. The brain computer interaction system associates the EEG signals to maneuver the robots. Video streaming with a night vision are provided so the user can interact freely with the surrounding.

In [30], an AR-based guiding system was proposed to enable new and inexperienced employees to accomplish a higher-level task with the assistance of an internal or external expert over video streaming. This work demonstrated potential for enhancement with 3D models and drawings to solve issues remotely and efficiently.

Previous work on UAV and HMD integration on video streaming was reported in |26|. The HMD is utilized in the system to provide virtual landmarks. These technologies assist UAVs in recognizing object-related commands even when they are not equipped with object detectors for a range of novel objects. With the integration of video streaming to their system, humans no longer need to be present in impossible or dangerous locations while piloting the UAV.

Another previous work in line with collaboration between UAV and HMD can be seen in [18]. These works combine multiple UAVs and multiple HMDs. HMDs are used to stream the view from multiple UAVs. With this, multiple HMD users can see multiple UAV views from each perspective of the UAV.

On the other hand, the proposed work investigates the use of Microsoft HoloLens as an HMD for improving HMI through a speech command system based on visualized video streaming. The proposed system uses a simple UAV speech command controller by which the UAV can easily understand the commands. Speech commands can be used for communication utilizing natural language processing.

\section{Proposed System Architecture}

The architecture of the proposed system is shown in Fig. 1. It, consists of an intractable MR application that fully controls and accesses the view from the UAV. In particular, two systems have been developed; a video streaming system, where the view from the UAV can be visualized on the MR device, and a UAV speech command and control system, where the MR device user can perform speech control on the UAV. The purposes of the proposed system is to enable the MR device user to observe the UAV view from video streaming and make decisions based on streaming to control the UAV using speech command controller. All the proposed work regarding with UAV control and UAV video streaming can be seen in Table. 1 .

Three devices, namely, the server, MR device, and UAV as clients, were included in the process to coordinate the entire systems. All the devices were connected to the same Wi-Fi connection as an access point to access the same access point. The detailed scheme for each system will further discussed and shown in Fig. 2 and Fig. 3 .

\subsection{Video Streaming System}

Fig. 2 shows the proposed video streaming system. To conclude the goal of the video streaming system, the MR device (HMD) as a client should access the UAV view. A specific UAV streaming IP address are available to access the UAV view. To get this specific IP address, since MR device and UAV are both clients that need to exchange data, they must be on the same connection. The connection details will be discussed later in Section IV. The main problem of having the same Wi-Fi connection is the connection range. This scheme is designed for indoor use in the current system. Hence, a wide-range network is not required. 
TABLE 1

Proposed Work Comparison with Existing Work

\begin{tabular}{|c|c|c|c|}
\hline Related Works & AR Devices & UAV Control & UAV Video Streaming \\
\hline Diego |31] & HoloLens & $\mathrm{O}$ & $\mathrm{X}$ \\
\hline Daniar & HoloLens & $\bar{X}$ & $\mathrm{O}$ \\
\hline Jeong [27] & HMD & $X$ & $\mathrm{O}$ \\
\hline Zhihao & HMD & $\bar{X}$ & $\mathrm{O}$ \\
\hline Sreeram [34] & Mobile Phone & $X$ & $\mathrm{O}$ \\
\hline Metehan $\mid \overline{35}]$ & $\mathrm{PC}$ & $X$ & $\mathrm{O}$ \\
\hline Thon $|3 \overline{3}|$ & PC & $\mathrm{X}$ & $\mathrm{O}$ \\
\hline Proposed System & HoloLens & $\mathrm{O}$ & $\mathrm{O}$ \\
\hline
\end{tabular}

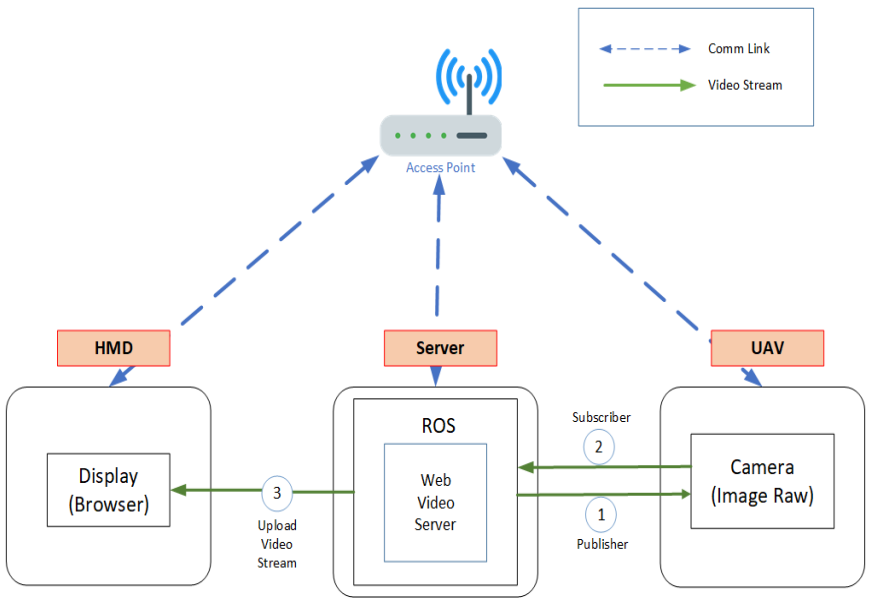

Fig. 2. Video streaming system model where HMD is streaming a video capture by UAV

To start the system, once both clients and server are connected, the server has to access a web_video_server package. This package are used to determine the IP address for video streaming before it can proceed. When the web_video_server is entirely operated, it requests raw image data from the UAV. After ROS subscribes to the UAV image raw data information, it is then forwarded to the web_video_server. The web_video_server will then broadcast the video streaming that will be accessible by the MR device when the user accesses the determined IP address from the web browser of the MR device. After accessing the UAV perspective, the MR device can confirm the UAV steering direction.

\subsection{Speech Command and Control System}

The speech command and control system scheme are displayed in Fig. 3 The speech command and control system when the speech command application on MR device is launched. To communicate between the server and all clients, ROSBridge are used. ROSBridge allows the ROS system, in here, server, and UAV, to connect to other systems through a WebScoket connection [37|. By communicating with the server, the ROSBridge for client is bridged to the ROSBridge for server. As soon as both connection are established, the server must launch the UAV driver. The movement script must be translated into a rostopic for UAV to move in compliance with the assigned rostopic. To translate the command to a rostopic, the process starts when the MR device user delivers a designed speech command

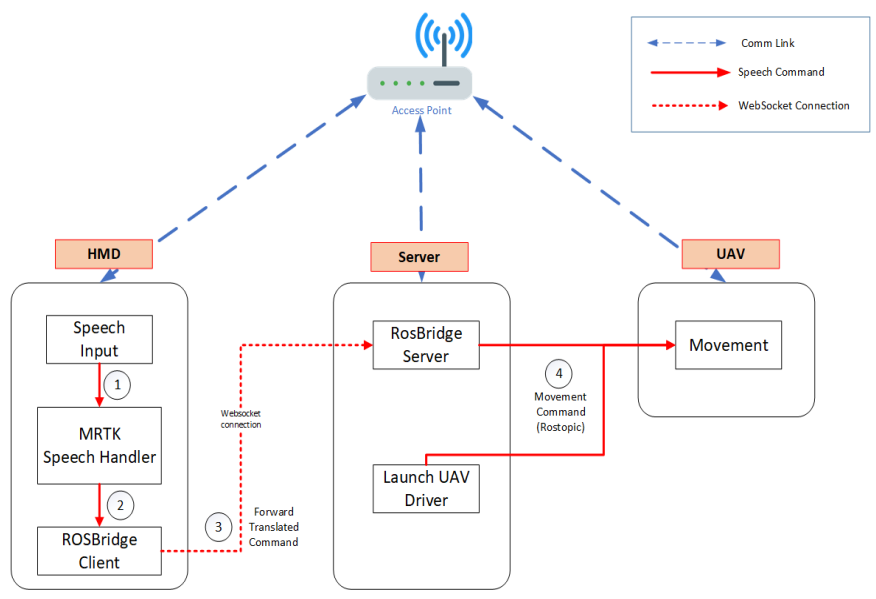

Fig. 3. Speech command\&control system model where speech input transmitted via ROSBridge

through the device. In here another tools are used, the tools is called as MRTK. Further details will be discussed in the next section. The MRTK "Speech Handler" package starts and recognizes commands. The successfully readable command will then be processed in NPM, which in NPM the detected speech command will translate it as a nodeJS format. However, if the command is unsuitable for the designed speech, the system will ignore the command.

Because it is connected to the server-side using the ROSBridge for server, the system will send the command to the ROSBridge for client after being converted to the nodeJS format. For the data connection between the ROSBridge client and the ROSBridge-Server to be efficiently transferred, ROSBridge must be running on the server-side. When the ROSBridge for server receives data, it must also operate the UAV driver node to connect to the UAV as a client. When a connection is established between the server and UAV, the saved data on the ROSBridge for server are processed by transforming the data into a rostopic. The rostopic will then be accessed, and the UAV will moves per the selected rostopic. The list of rostopics and commands can be seen in Table 5

\section{IMPLEMENTATION}

Once the system analysis has been completed and all proposed diagrams have been created, this section will go through the implementation component in depth. For developing the application, since the devices have different operating system, each device implementation is done first 
before it is combined with each devices. An architecture diagram is made to explain the development process. The development architecture diagram can be seen in Fig. 4 The development process is classified in two device-based system; Universal Windows Platform (UWP) for Microsoft HoloLens, and ROS for server and Bebop 2. Details for each device development needs can be seen in the subsection 4.1 and 4.2. The whole process conducted by developing for each system, then, combine everything and setup the network connection and device-to-device (D2D) communication. Details of the connection and communication can be seen in subsection 4.3 and the user interface for Microsoft HoloLens details were explained in subsection 4.4.

The implementation were conducted using Microsoft HoloLens as the MR device, a server, and a Bebop 2 as the UAV. The HoloLens will emphasize on how control is optimized and how streaming from Bebop 2 is displayed as a holograms. The middleware between HoloLens and Bebop 2 is the server. Bebop 2 will be the client that can move in response to the movement of another client.

The HoloLens specifications can be seen in Table 2 The HoloLens is a HMD which includes a head-tracking sensor using four visible light cameras, eye-tracking sensors using two infrared cameras, an 8-megapixel camera, a five-channels microphone array, a built-in spatial sound for speakers, an inertial measurement unit with an accelerometer, gyroscope, and magnetometer, and an ambient light sensor. Furthermore, the Microsoft HoloLens system on chip stores the CPU and GPU, including a Qualcomm Snapdragon 850 and a second-generation Microsoft custommade holographic processing unit capable of processing and integrating data from all available sensors and performing tasks such as spatial mapping, tracking, gesture recognition, and voice and speech recognition.

The proposed work uses a laptop-based server, that uses Lenovo T580. The laptop specification can be seen in Table 3 With an 8th generation i7 CPU and NVIDIA MX150 GPU, a 32GB memory with a Ubuntu-based operating system with 16.04.7 LTS version. In the server, a ROS Kinetic 16.04 was installed to process the entire system. The server component is utilized to integrate data into Bebop 2 and merge data from Microsoft HoloLens. Weighted around 1,97kg make this server easy to use and comfortable. The laptop was used to activate the rosbridge, bebop_driver, and web_video_server.

TABLE 2

Microsoft HoloLens Specification

\begin{tabular}{|c|l|}
\hline Microsoft HoloLens & \multicolumn{1}{|c|}{ Specification } \\
\hline Operating System & Windows Core OS \\
\hline Processor & Qualcomm Snapdragon 850 \\
\hline Memory & 4-GB LPDDR4x system DRAM \\
\hline Storage & 64-GB UFS 2.1 \\
\hline Display & See-through holographic lenses \\
\hline Sound & Built-in spatial sound \\
\hline \multirow{2}{*}{ Input } & Eye tracking \\
\cline { 2 - 2 } & Spatial tracking \\
\cline { 2 - 2 } & Hand tracking \\
\hline Camera & 8 MP 1080P30 video \\
\hline \multirow{2}{*}{ Connectivity } & Bluetooth LE 5.0, 802.11 2x2 \\
\cline { 2 - 2 } & Wi-Fi 802.11 ac 2×2 \\
\hline
\end{tabular}
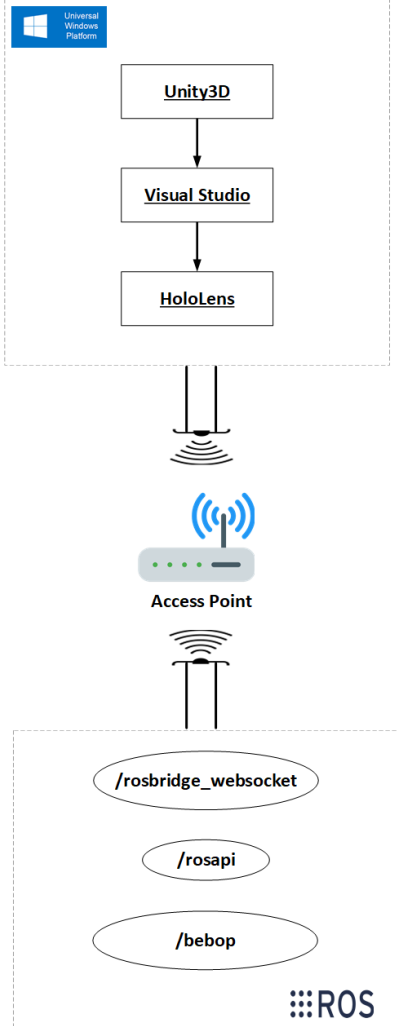

Fig. 4. The development architecture diagram where MRTK communicate with ROS

TABLE 3

Server Specification

\begin{tabular}{|c|l|}
\hline Lenovo T580 & \multicolumn{1}{|c|}{ Specifications } \\
\hline Operating System & Ubuntu 16.04.7 LTS \\
\hline CPU & Qualcomm Snapdragon 850 \\
\hline Memory & 32-GB LPDDR4x system DRAM \\
\hline Storage & 256GB SSD \\
\hline \multirow{2}{*}{ Connectivity } & Bluetooth 4.1, M.2 card \\
\cline { 2 - 2 } & Wi-Fi 2x2 802.11ac \\
\hline Battery & $12.5 \mathrm{hr}$ \\
\hline
\end{tabular}

The other client, Parrot company Bebop 2 is a low-cost, small-scale unmanned aerial vehicle. Parrot Bebop 2 specification can be seen in Table 4 It is the next generation of ready-to-fly, stable, and safe multi-rotor micro-class UAVs, following AR Drone 2.0. The Parrot Bebop 2 power comes with a wide-angle custom lens and a distortion-preventing system, as well as 3-axis stabilization. The resolution of the video recording was 1920 x 1080 (1080p). The following features are used to capture images: $14 \mathrm{MP}$ sensor resolution, CMOS sensor that allows full HD shooting at $30 \mathrm{fps}$, 4095 x 3072 picture resolution, JPG, RAW, and DNG image generation. The UAV had a flight time of approximately 25 min. Bebop 2 runs on UNIX-based BusyBox, and is powered by a P7 dual-core CPU, a quad-core GPU, and 8 GB of RAM.

\subsection{HoloLens Development Software}

Development software is needed to create the whole system on the Microsoft HoloLens. Unity3D [38] is a popular development tool for create HoloLens applications. Previously, Unity3D was widely known as a game engine with 
TABLE 4

Parrot Bebop 2 Specification

\begin{tabular}{|c|l|}
\hline Parrot Bebop 2 & \multicolumn{1}{|c|}{ Specification } \\
\hline Hardware & CPU Dual-core A9 \\
\hline Memory & 8GB Internal memory \\
\hline \multirow{2}{*}{ Battery } & 2700mAh \\
\cline { 2 - 2 } & 3 cells lipo-battery (Typ. 15 minutes) \\
\hline Rotors & Motor 1280kv \\
\hline Video \& Picture & 14 MP wide-angle, full HD 1080p video \\
\hline \multirow{4}{*}{ Sensors } & Ultrasound (6 Meters) \\
\cline { 2 - 2 } & Barometer (20cm Precision) \\
\cline { 2 - 2 } & Altimeter \\
\cline { 2 - 2 } & Hi-speed vertical camera \\
\hline \multirow{3}{*}{ Wi-Fi \& Transmission } & Access point network \\
\cline { 2 - 2 } & Wi-Fi AC-type \\
\cline { 2 - 2 } & 2bi-band antennas (2,4 and 5 GHz) MIMO \\
\hline GPS & Built-in GPS \\
\hline Software & Linux and SDK \\
\hline
\end{tabular}

numerous modules. As development software improves, Unity3D is now known as a cross-platform development software capable of creating VR, AR, and MR applications. Some platforms are available for development, such as the Windows platform, Mac OS and Android. Unity3D is used on the system because the proposed system is an MR-based system under a universal windows platform, supported by Unity3D.

Some notable aspects of Unity3D are its scripting capabilities. Unity3D supports JavaScript, C\# as their primary scripting language; it can also be extended as well with VB.net, VB6, Delphi, and more. These script are combined with .NET libraries to supports XML files, databases and networking. Scripts in Unity3D are compiled into native code and run nearly as fast as C++. It is easy to implement and use, with quick iteration times. The ease of use is ideal for scripting languages; additionally, these languages can be mixed and matched. The Unity3 $\mathrm{D}$ game engine and developer environment are tightly integrated, providing a capable visual editor and network language levels.

Additional HMD development tools were used in this study. These tools are used to handle specifics purposes while developing applications. This study uses the tools to develop the speech input handler and UI handler before deploying the application on the HoloLens device. These tools are called as Mixed Reality Tool-Kit (MRTK) [40]. MRTK was used as the primary speech input handler and the UI handler.

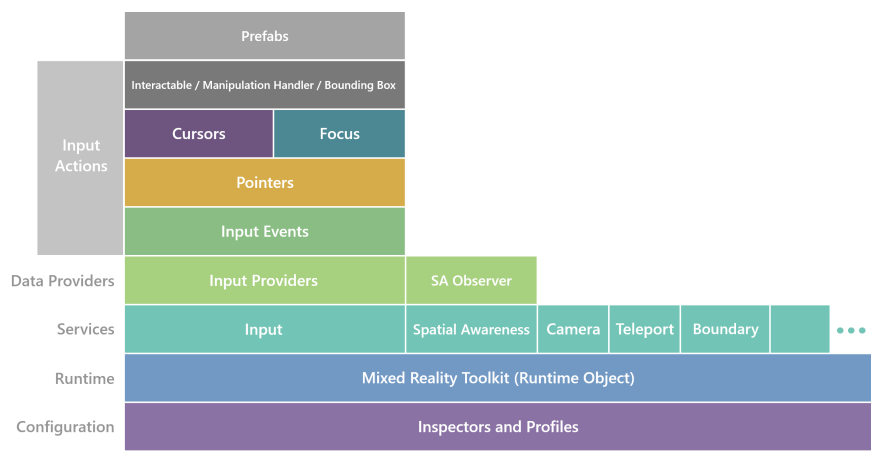

Fig. 5. MRTK Architecture Overview 39]
The MRTK is a library of scripts and components developers may use to build applications for Microsoft HoloLens and other Windows Mixed Reality headsets. The details of MRTK structure can be seen in Fig. 5 MRTK contains configuration, runtime, service, data providers and input action. The configurations are designed which users can modify as what they want by configuring the profiles, the profiles are used to configure the behavior of the corresponding subsystems. Runtime object are designed to manages life and runtime of its services. Services are the main part of the MRTK. Inputs are designed to collaborate with the profile to provides the main entry point for the Toolkit in the project. User can modify the input that the user needs, in this paper are speech input and UI. Data providers are managing the corresponding data to a particular source of input. While the input itself are the main interaction between the user and the application. The user can use intractable, cursor, focus, pointer, controller to interact with the application.

This subsection will only explain the use of the MRTK speech input handler, while MRTK UI handler will be discussed in the following subsection. In this study, the MRTK speech input handler handles the HoloLens user input as a voice command as the services part. It translates the voice command as data that can be forwarded so that the server can obtain the input data from the HoloLens user.

\subsection{Server and Bebop 2 Development Kit}

The robot operating system (ROS) has gained much popularity for developing robotic systems inside the laboratory and, in the industry. The ROS is a flexible open-source system that operates a robot [41]. It includes a set of tools, a library, and packs that help to simplify the operation of a robust robot. Controlling code in robot operating systems can also be written in a more abstract language, allowing it to be easily adapted to a different controlling microprocessor. ROS can adapt to many types of robots and unmanned vehicles, such as unmanned aerial vehicles (UAVs), unmanned ground vehicles (UGVs), unmanned surface vehicles (USVs), unmanned underwater vehicles (UUVs) and more. ROS are utilized in the system because they are simple to use, adaptive, easy to install, and maintain, and can be modified to meet the systems needs.

The proposed system uses ROS [42] for implementation. Some ROS resources that must be accessed for the proposed system are the ROS package and message types. To process data from the server to the Bebop 2 client, rosmaster, rosnodes, rostopics, and rosmessages are required. For implementation in video streaming systems, rostopics called web_video_server are accessed. Which topics will manage the video subscribing and publishing from Bebop 2 to the server and vice versa. For implementation in a control system, the accessed rostopics depend on the control. The details of the control are presented in Table 5 Based on the table, to make the Bebop take off, a bebop_takeoff topic is accessed. To make the Bebop land, a bebop_land is accessed. Meanwhile, for the Bebop 2 movement, bebop_twist is accessed. These three topics have their rosmessages; rosmessages are data structures to communicate between rosnodes. 
TABLE 5

ROS Topics and Command List

\begin{tabular}{|c|c|c|}
\hline Movement & Speech & ROS Topics \\
\hline Take Off & Take Off & Bebop/Takeoff \\
\hline Landing & Land & Bebop/Land \\
\hline Front & Go Front & \multirow{4}{*}{ Bebop/Twist } \\
\hline Back & Go Back & \\
\hline Left & Go Left & \\
\hline Right & Go Right & \\
\hline
\end{tabular}

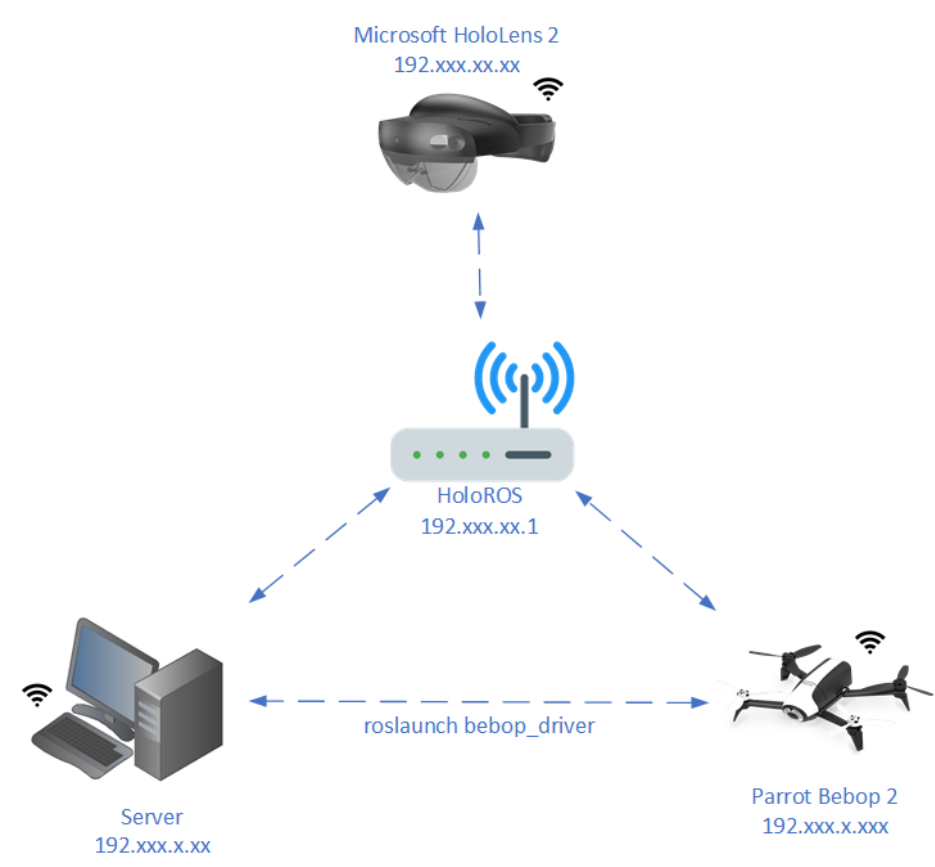

Fig. 6. The Communication link of the proposed system model. All devices connected to one single Gateway

\subsection{Connectors and Communication}

This connectors and communication implementation section focuses on the ROS and Unity3D connectors and network communication. Connectors are a crucial component of the implementation. This section focuses on connecting the application inside the client device HoloLens, with the server as a rosmaster to deliver commands to the UAV. ROSBridge is the middleware used in the proposed system. ROSBridge is a middleware that communicate ROS- based programs and non-ROS-based programs. ROSbridge consists of a JSON-based API to access ROS functionality so that the nonROS-based programs can communicate and interact with ROS-based programs, such as the server.

ROSBridge uses a WebScoket connection, which operates in a single TCP/IP socket. WebSocket can support upstream and downstream communications over a single connection. ROSBridge is divided into two device needs: ROSBridgeServer and ROSBridge-Client. The ROSBridge-Client and the ROSBridge-Server are set up to be connected in the Unity3D application before deploying the application on the HoloLens. The ROSBridge-Server is installed on the server. Because the programming languages are different, ROS-Sharp is used to connect them. Python and $\mathrm{C}++$ are utilized in the server, whereas C\# and JavaScript are used in Unity3D.
When creating an implementation-based system with several clients, the communication component must be considered. The features of the communication link are shown in Fig. 6. In this study, an Internet connection is not required. However, $\mathrm{Wi}-\mathrm{Fi}$ as an access point connection is used to connect all devices. The gateway of the access point connection was 192.168.0.*. After HoloLens and the server were connected to the same access point, Bebop 2 was connected to the preferred access point. Bebop 2 generally has its separate access point, used by the client to access the Bebop 2. This does not apply to the system, because the current system as it requires multiple clients. In this case, IP pushing towards the Bebop is required, and Bebop 2 can be connected to the preferred access point. IP pushing is used to make the Bebop accessible without making Bebop an access point.

\subsection{User Interface}

The user interface is a visual representation of the application that the user sees. User interface (UI) design has increased in popularity over the years, becoming one of the most creative, innovative, and fascinating fields in technological innovations. The UI was created using the Unity3D 2019.4.12f1 version in collaboration with the MRTK, as explained in the previous subsection. The UI is divided into video streaming UI and speech command \& control UI. The general UI can be seen in Fig. 7 The real video streaming UI can be seen in Fig. 7(a) when the bebop is on land and Fig. 7(b) when the bebop is flying. The designed speech command \& control UI can be seen in Fig. 7(c) and the real one can be seen in Fig. $7(\mathrm{~d})$.

In the proposed system, the UI is made using holograms created by MRTK. MRTK is used as the primary speech input handler as well as the UI handler. The MRTK UI handler, combined with spatial mapping, gaze handlers, and gesture handlers, is used to construct the UI. The controller interface is automatically identified in the user real environment through spatial mapping and gaze and gesture handlers allow the user to interact with the application. All scripting will be handled in Visual Studio 2019 before the implementation of the GUI system. The script can then be assigned to the button in Unity3D. The system could be built and deployed on HoloLens once all of the system components were created. The deployment procedure in Visual Studio 2019 must be completed before being shown on the HoloLens device as an application.

\section{EXPERIMENT AND Result}

An experiment on the development of MR as a GCS for UAVs was conducted. The experiment only considered devices that support the TCP protocol. The TCP protocol is used to calculate the throughput, round-trip time (RTT) and latency for video streaming (VS) and combined video streaming and command and control systems (VSC\&C). Video streaming is uploaded as a hypertext transfer protocol (HTTP) from the server, which is why the HoloLens access it as a TCP protocol. A total of 20 trials were conducted for each scenario. A speech accuracy analysis was also performed to assess the users' speech capabilities using 


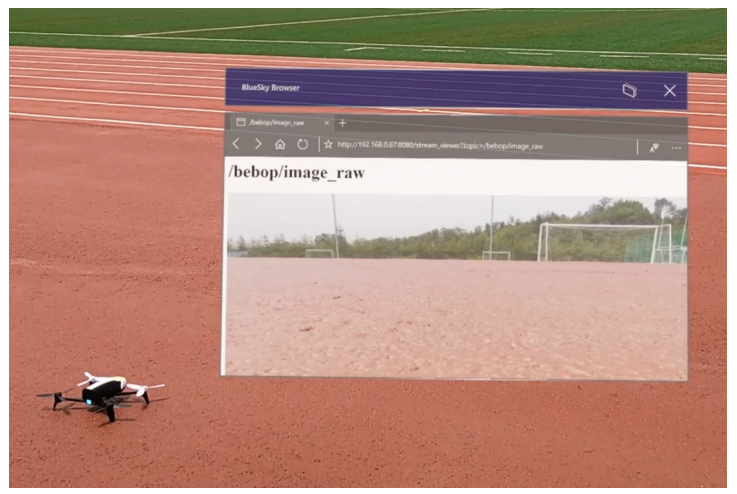

(a)

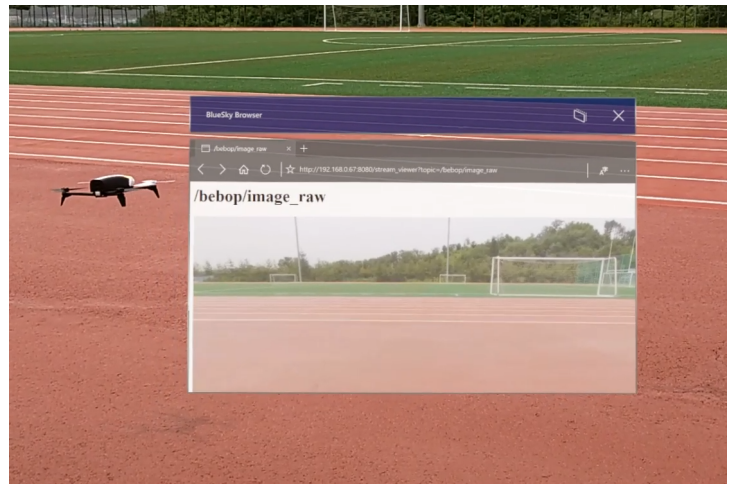

(b)

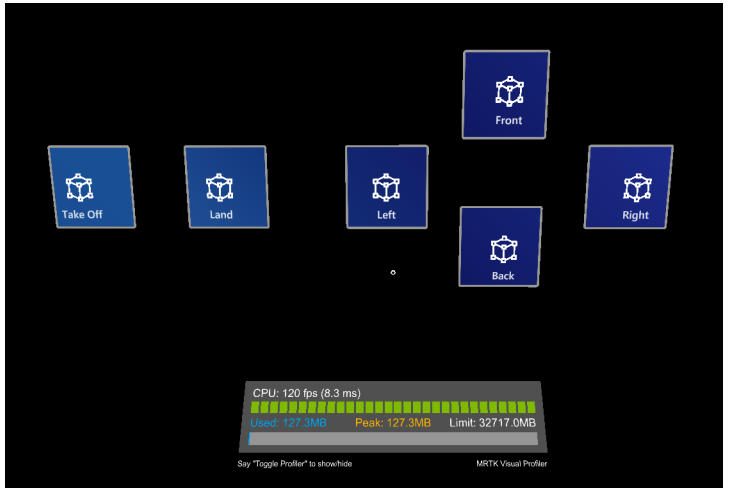

(c)

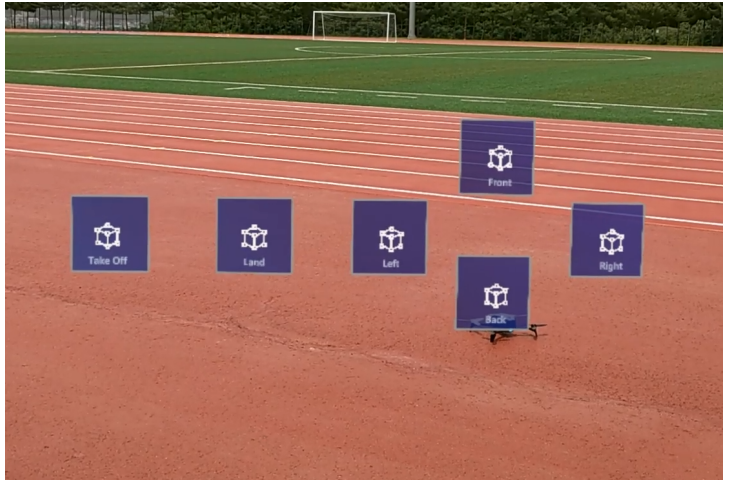

(d)

Fig. 7. (a) HoloLens Video streaming view when UAV still on initial position, (b) HoloLens Video streaming view when UAV Take Off, (c) Designed Speech Command \& Control User Interface, and (d) Real Speech Command \& Control User Interface

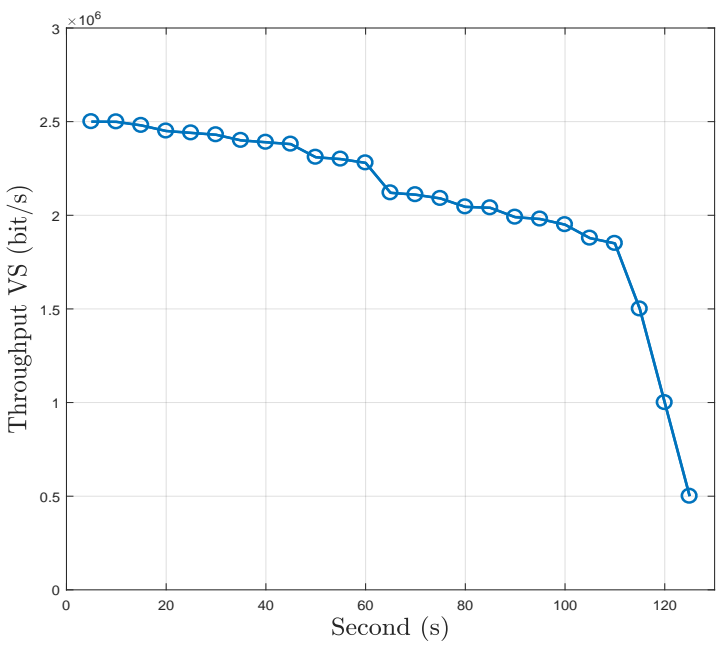

Fig. 8. Server to HoloLens Video Streaming Throughput

the application. The experiment for command and control $(C \& C)$ will not be counted because the protocol utilized is UDP.

First, for the VS-only performance evaluation, a 5 second interval sampling period is utilized, followed by a total length of $125 \mathrm{~s}$ for each $i$ trial. Second, for the combined VS and C\&C performance evaluation, the user was given a single command to the UAV with a period of 15 seconds, and a video was streamed simultaneously. Therefore, RTT and throughput were sampled every $15 \mathrm{~s}$ for $600 \mathrm{~s}$ of total observation time per $i$ trial. Finally, to count the latency for VS and VSC\&C, servers are used to count the data transfer transmission. When the user gives a single command to the $\mathrm{UAV}^{1}$, the server checks the latency time for both UAV and the HoloLens. The server will count the time process from each device nodes and combined them and calculated the average value as an average end-to-end latency.

\subsection{Throughput}

Throughput is the average rate at which data are obtained by a node over a given measurement interval. Throughput measurements are conducted in the VS only and VSC\&C scenarios. Equation 11 was used to measure the system throughput in both cases.

$$
\chi=\frac{N^{r x}}{t},
$$

where $N^{r x}$ is the received packet for each node and $t$ is the time(s) that is received from the total packet trials.

The first scenario for throughput performance was the VS system. The trials were conducted 20 times. Every trial was conducted with $125 \mathrm{~s}$ of simultaneous streams. Fig. 8 shows that the maximum throughput was observed at $5 \mathrm{~s}$ intervals which reached $2.5 \times 10^{6} \mathrm{bit} / \mathrm{s}$ and the minimum was at $125 \mathrm{~s}$ intervals which was $0.5 \times 10^{6} \mathrm{bit} / \mathrm{s}$.

1. C\&C system required 344 bits of packet size for each command. Video streaming required roughly 7388.56 bits for VS only and 12098.16 bits for VSC\&C for every $i$ th trial 
The second throughput performance scenario was for VSC\&C. The executed commands are listed in Table 5 The commands are given in 15 second interval sampling period. The results of the VSC\&C throughput sampling scenario are shown in Fig. 9. The maximum throughput was observed in $15 \mathrm{~s}$ interval, which reaches $3.25 \times 10^{6} \mathrm{bit} / \mathrm{s}$, with the minimum at $600 \mathrm{~s}$ which is $1.3 \times 10^{6} \mathrm{bit} / \mathrm{s}$.

In general, all the throughput figure in Fig. 9 and Fig. 8 shown that throughput is lower for the time being. The decrements occur since all the device have been processed all the data. The throughput is gradually decreases with regarding time because the devices are processing the data concurrently.

\subsection{Round-time Trip (RTT)}

The round-time trip is the time it takes for a signal to be transmitted plus the time it takes for that signal to be acknowledged as having been received. RTT measurements combined transmission and acknowledgment for both servers and HoloLens. RTT measurements were conducted in the VS and VSC\&C scenarios.

The first performance scenario for the RTT was the VS system. The experiments were conducted in 20 trials. Each trial was performed for a total of $125 \mathrm{~s}$ of stream time. Fig. 10 shows that the RTT for VS has the maximum RTT at 250s intervals, which is $0.128 \mathrm{~s}$. The minimum RTT was observed to be $0.04 s$, and was seen in 10s intervals. The average RTT was calculated using the following equation:

$$
\overline{\mu_{x}}=\frac{1}{T} \sum_{s_{x}=0}^{T} \mu\left(s_{x}\right)
$$

where $T$ denotes the total observation time, $s$ denotes the second interval, and $\mu_{x}$ denotes the RTT obtained on the $s^{t} h$ interval for the $x: V S \operatorname{or} V S C \& C$ scenario. With $T=125$ and $s_{V C}=[0,5,10 \cdots, T]$, the values obtained from Eq. (2) for $\overline{\mu_{V S}}$ is $0.073 \mathrm{~s}$.

The second RTT performance scenario was for the VSC\&C. The results of the second scenario are shown in

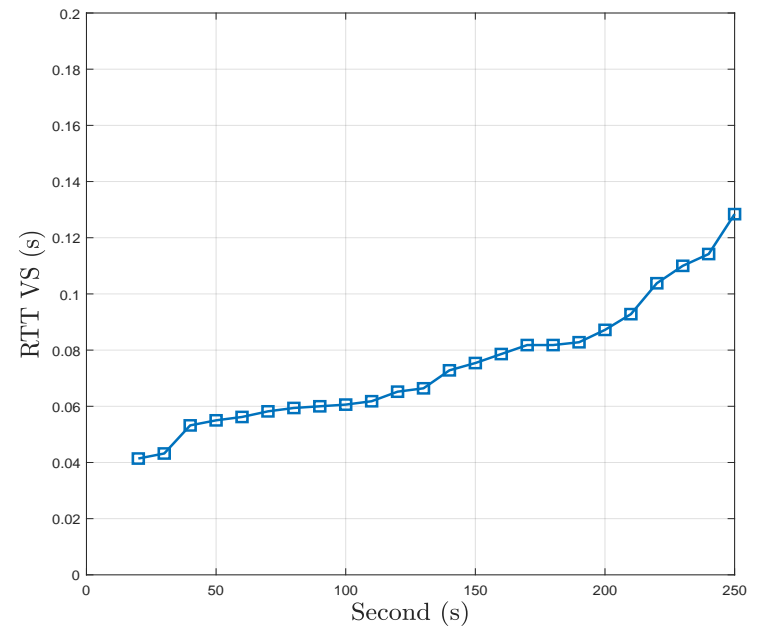

Fig. 10. Server to HoloLens Video Streaming RTT

Fig. 11 The results shown that the VSC\&C RTT has $0.182 \mathrm{~s}$ as the maximum RTT which occured in 250s. The minimum RTT can be seen in the $15 \mathrm{~s}$ interval, which is $0.001 \mathrm{~s}$.

To measure the average RTT for VSC\&C, Eq. (2) was used. Where the $T=600$ and $s=[0,15,30 \cdots, T]$. The value obtained for $\overline{\mu_{V S C \& C}}$ was $0.063 \mathrm{~s}$.

In general, all the RTT figure in Fig. 10 and Fig. 11 shown that RTT is increased for the time being. The increments occur since the server response time is high, since server not only handle the request of each device for video streaming, and command and control. Increased RTT is also caused by packet data, as video streaming requires larger packet sizes than command and control, resulting in a higher RTT for combined video streaming and command and control systems.

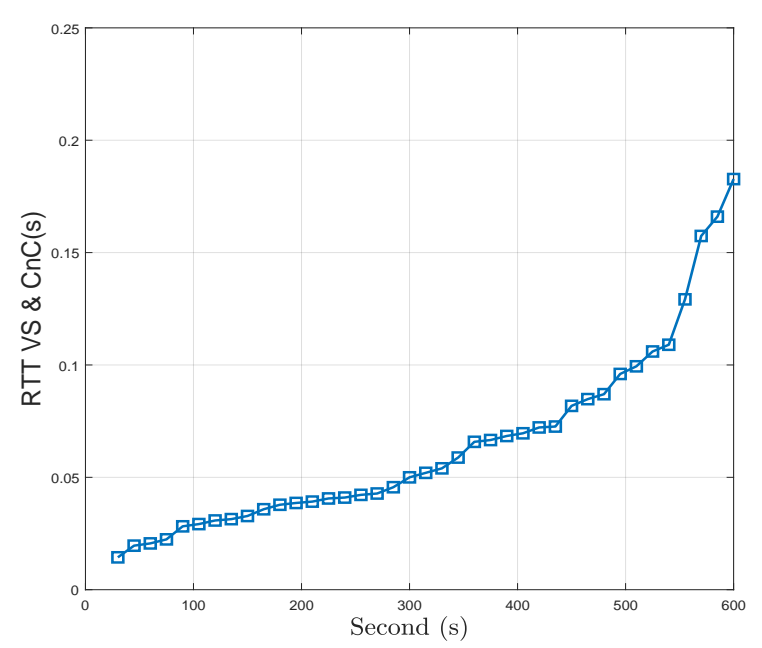

Fig. 11. Server to HoloLens Video Streaming and Command \& Control RTT
Fig. 9. Server to HoloLens Video Streaming and Command \& Control Throughput

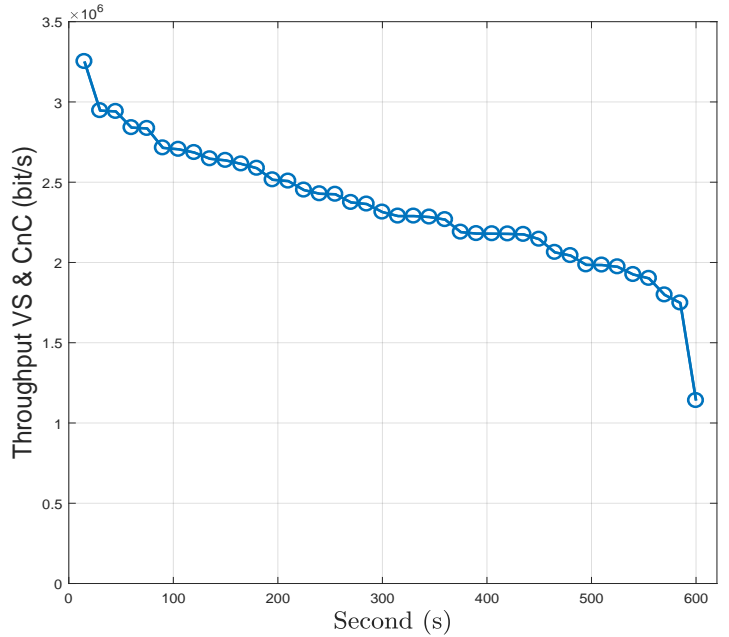


TABLE 6

Total Average end-to-end latency result for each $i$ trials

\begin{tabular}{|c|c|c|}
\hline Trials & Video Streaming (s) & $\begin{array}{c}\text { Video Streaming and } \\
\text { Command \& Control (s) }\end{array}$ \\
\hline 1 & 0.054 & 0.107 \\
\hline 2 & 0.055 & 0.112 \\
\hline 3 & 0.040 & 0.152 \\
\hline 4 & 0.047 & 0.139 \\
\hline 5 & 0.059 & 0.168 \\
\hline 6 & 0.054 & 0.151 \\
\hline 7 & 0.037 & 0.161 \\
\hline 8 & 0.038 & 0.153 \\
\hline 9 & 0.057 & 0.189 \\
\hline 10 & 0.059 & 0.163 \\
\hline 11 & 0.061 & 0.165 \\
\hline 12 & 0.064 & 0.143 \\
\hline 13 & 0.048 & 0.169 \\
\hline 14 & 0.038 & 0.179 \\
\hline 15 & 0.034 & 0.189 \\
\hline 16 & 0.050 & 0.151 \\
\hline 17 & 0.036 & 0.139 \\
\hline 18 & 0.042 & 0.195 \\
\hline 19 & 0.053 & 0.182 \\
\hline 20 & 0.039 & 0.178 \\
\hline
\end{tabular}

\subsection{Latency}

The end-to-end network latency is a packet delay from one client to another client. In this study, it was determine by the following relation: $L=\left\|t_{o}-t_{1}\right\|$. Here, $t_{o}$ denotes the time of the network packet departing from HoloLens, and $t_{1}$ denotes the time of packet arrival on the UAV. Latency measurements were also performed in the VS and VSC\&C scenarios.

Fig. 12 represents the latency that occurred in VS and VSC\&C scenarios. Experiments were conducted in a total of 20 trials. By counting the average for a total of $i 125$ seconds for VS, and 600 seconds for VSC\&C scheme. In VS latency performance measurement, the minimum average latency was $0.034 s$ on trial 15th, while the maximum average latency was $0.064 \mathrm{~s}$ in trial 12 th. The total average latency for VS measurement was $0.048 s$. For VSC\&C latency measurement, the minimum latency was at trial 1st which gain $0.107 \mathrm{~s}$ and the maximum latency is at trial 18th with $0.195 \mathrm{~s}$. The total average latency for VSC\&C measurement was $0.159 \mathrm{~s}$.

To conclude, Fig. 12 combine all the average latency for every trials. Each $i$ trials average amount can be seen in Table. 6. The minimum amount for the whole VS system occur in trial 15 which has $0.034 \mathrm{~s}$ and the maximum amount can be seen in trial 12 as $0.064 s$. For VSC\&C system, the minimum average latency occur in the 1st trial which has $0.107 \mathrm{~s}$ and the maximum amount is 0.195 which occur in trial 18th.

\subsection{Speech Accuracy Test}

In this section, an analysis of the system performance is presented. The results of the performance tests are the number of retries and the number of errors that occur while giving speech commands in Table 5 to the UAV. Here, it is presumed that the total number of trials $I$ is 20 , where every $i^{\text {th }}$ trial contains 102 random speech commands from
TABLE 7

Speech Accuracy Error and Retries Counts for $i$ th trial

\begin{tabular}{|c|c|c|}
\hline Trials & Error (\%) & With Retries (\%) \\
\hline 1 & 8.82 & 20.93 \\
\hline 2 & 3.92 & 9.73 \\
\hline 3 & 5.88 & 13.56 \\
\hline 4 & 3.92 & 7.27 \\
\hline 5 & 2.94 & 5.56 \\
\hline 6 & 2.94 & 5.56 \\
\hline 7 & 2.94 & 5.56 \\
\hline 8 & 0.00 & 0.00 \\
\hline 9 & 0.98 & 1.92 \\
\hline 10 & 1.96 & 4.67 \\
\hline 11 & 3.92 & 8.93 \\
\hline 12 & 1.96 & 2.86 \\
\hline 13 & 2.94 & 4.67 \\
\hline 14 & 2.94 & 5.56 \\
\hline 15 & 0.98 & 0.97 \\
\hline 16 & 0.00 & 0.00 \\
\hline 17 & 0.98 & 1.92 \\
\hline 18 & 1.96 & 2.86 \\
\hline 19 & 0.00 & 0.00 \\
\hline 20 & 2.94 & 3.77 \\
\hline
\end{tabular}

Table 5 Additionally, to achieve reliability of the system, it is considered that the fail threshold is $\epsilon_{x} \approx 0.9 \times$ Trials ${ }^{2}$

Fig. 13 represents the number of $\xi_{c}$ of each $i^{t h}$ trial, where $\xi_{c}$ denotes number of fail counts. The fail counts are calculated when UAV action is not suitable with the speech command. It is considered that the system is considered as reliable, since the fail counts from all $i$ trials fall below $\epsilon_{c}$ 3 Additionally, several indexes of $i$ had similar fail counts, which are 3 to 4 errors. Fig. 14 shows the total number of speech command retries that have to be made until the UAV takes action based on it, and it is denoted by $\xi_{e}$. The highest retry count was on $i=1$ with the a total of 27 retry. Based on this condition, it can be concluded that the average number retries of attempts to be performed is $\approx \frac{102}{27} \approx 3$.

2. The $\epsilon_{x}$ represents threshold for different scenarios, and Trials is the total trial test performance.

3. Obtain fail count threshold with calculation $\epsilon_{c} \approx 0.9 \times 102 \approx 90$.

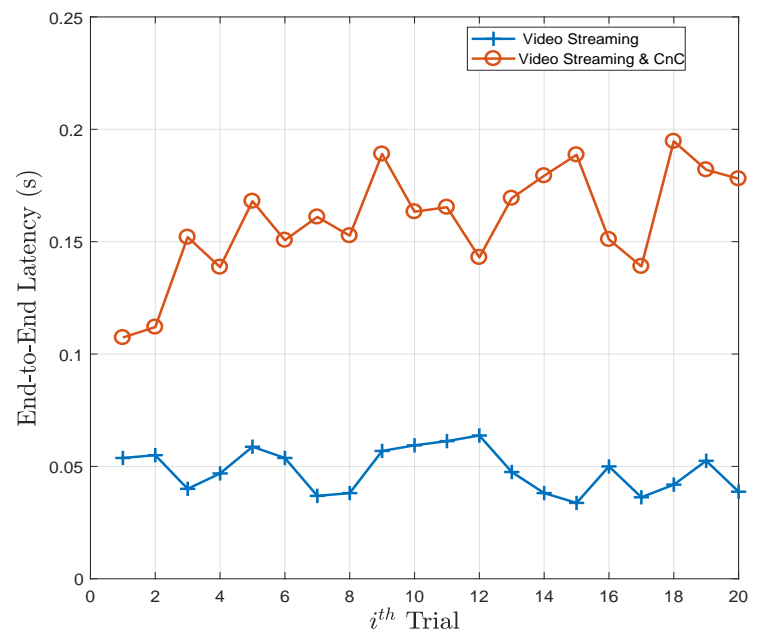

Fig. 12. The result of end to end average latency devices with $I=20$ 


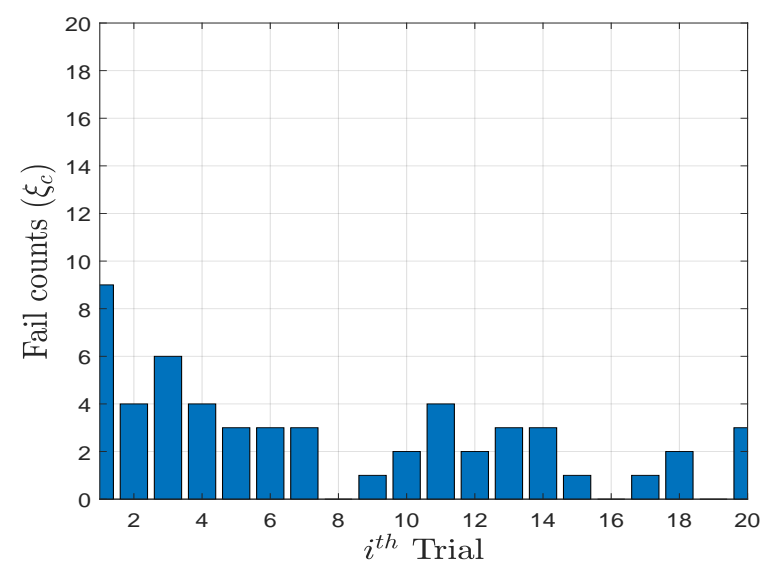

Fig. 13. Number of wrong actions that UAV takes while receiving speech command

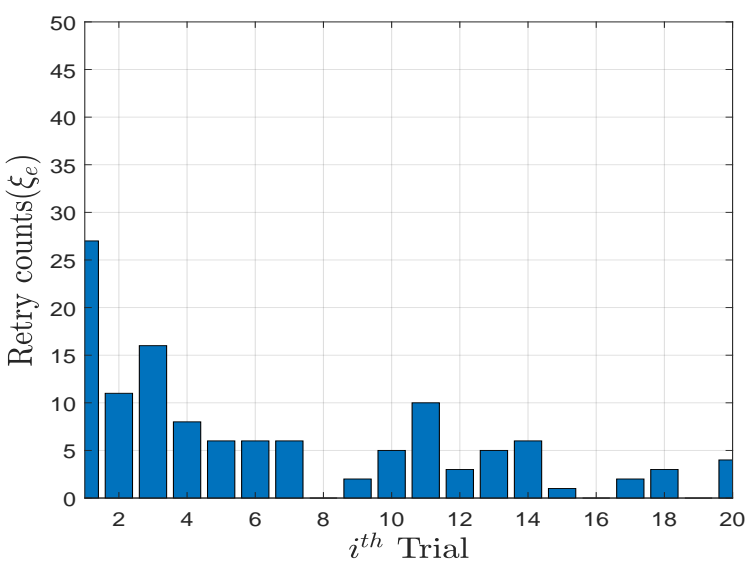

Fig. 14. Number of retry command that has to be repeated in order to obtain correct UAV action

$$
\text { SuccessRate }=1-\frac{E}{\text { TotalTrial }} X 100 \%,
$$

where success rate is the percentage of attempts that result in success. Eq.(3) is used to determine the success rate of the user interface. Where $E$ is the error trial amount, and the total trial is the total of the command trial, which is a 2,040 command.

All total of the $i$ trial result can be seen in Table. 7 From total of $20 i$ th trial, $3 i$ th trial defined as perfect with no occurred error and retries counts. Total for $20 i$ th trial average for error counts was $2.65 \%$, the average retries counts was $5.31 \%$. Three trials were having $100 \%$ success rate, which mean there is no occurred error and retries.

\section{Conclusion}

This study describes the use of MR as UAV ground control station. Real implementation and demonstration for such a system consisting of a speech command control (C\&C) and video streaming (VS) systems have been performed. The system consist of a Server, Microsoft Hololens as an HMD and Parrot Bebop 2 as the UAV. The experiment is conducted by measure the throughput, RTT, and latency.
The experimental calculation is focused only on the TCP protocol because of the different protocols used in the system. The calculation focuses on throughput, RTT, and latency for VS and VSC\&C and the speech accuracy for the speech command system.

Based on the throughput results for VS and VSC\&C the throughput for VSC\&C is considered as stable based on the increment for each interval. The maximum throughput amount is $3.25 \times 10^{6} \mathrm{bit} / \mathrm{s}$ for VSC\&C and $2.5 \times 10^{6} \mathrm{bit} / \mathrm{s}$ for VS. In addition, comparison for RTT graph also has been done. For VS, the minimum RTT is $0.04 s$ and the maximum RTT is 0.128 s. In VSC\&C measurement, the minimum RTT is $0.001 s$ and the maximum RTT is $0.182 s$.

The latency result shows that the minimum VS latency performance is $0.034 \mathrm{~s}$, while the maximum latency is $0.064 \mathrm{~s}$. In the VSC\&C latency measurement, the minimum latency is $0.107 \mathrm{~s}$ and the maximum latency is $0.195 \mathrm{~s}$. The speech command accuracy test was also conducted by counting the Fail and Retry counts. The system is considered reliable for speech system because the fail counts fall below the threshold. Meanwhile, the retry count for the speech accuracy test is $\approx \frac{102}{27} \approx 3$.

In the future, improvements in video streaming are needed. In addition, another type of communication such as 5G LTE connection should be added to improve the throughput, RTT and latency in the current system. Finally, more manuscripts will be prepared to provide more details on this work.

\section{ACKNOWLEDGMENT}

This research was supported by the MSIT(Ministry of Science and ICT), Korea, under the Grand Information Technology Research Center support program (IITP-2021-20200-01612) supervised by the IITP (Institute for Information \& communications Technology Planning \& Evaluation)

\section{REFERENCES}

[1] D. Zuehlke, "Smartfactory-towards a factory-of-things," Annual reviews in control, vol. 34, no. 1, pp. 129-138, 2010.

[2] J. Lee, H. Davari, J. Singh, and V. Pandhare, "Industrial artificial intelligence for industry 4.0-based manufacturing systems," Manufacturing Letters, vol. 18, pp. 20-23, 2018.

[3] J. Wan, S. Tang, Q. Hua, D. Li, C. Liu, and J. Lloret, "Contextaware cloud robotics for material handling in cognitive industrial internet of things," IEEE Internet of Things Journal, vol. 5, no. 4, pp. 2272-2281, 2018.

[4] M. Gattullo, G. W. Scurati, M. Fiorentino, A. E. Uva, F. Ferrise, and M. Bordegoni, "Towards augmented reality manuals for industry 4.0: A methodology," Robotics and Computer-Integrated Manufacturing, vol. 56, pp. 276-286, 2019.

[5] D. Gorecky, M. Schmitt, M. Loskyll, and D. Zühlke, "Humanmachine-interaction in the industry 4.0 era," in 2014 12th IEEE International Conference on Industrial Informatics (INDIN), 2014, pp. 289-294.

[6] T. Sutar and S. Pawar, "Smart glasses: Digital assistance in industry," in Advances in Signal and Data Processing, S. N. Merchant, K. Warhade, and D. Adhikari, Eds. Springer Singapore, 2021.

[7] "Microsoft hololens 2," Accessed: May. 25, 2021. [Online]. Available: https://www.microsoft.com/en-us/hololens/hardware

[8] A. Sun, J. Shi, and W. Li, "A precise fingertip tracking algorithm based on 3d motion mapping for hololens," in 2019 11th International Conference on Intelligent Human-Machine Systems and Cybernetics (IHMSC), vol. 1, 2019, pp. 229-232. 
[9] L. F. Luque Vega, E. Lopez-Neri, C. A. Arellano-Muro, L. E. González-Jiménez, J. Ghommam, and R. Carrasco-Navarro, “Uav flight instructional design for industry 4.0 based on the framework of educational mechatronics," in IECON 2020 The 46th Annual Conference of the IEEE Industrial Electronics Society, 2020, pp. 23132318.

[10] J. G. Martinez, M. Gheisari, and L. F. Alarcón, "Uav integration in current construction safety planning and monitoring processes: Case study of a high-rise building construction project in chile," Journal of Management in Engineering, vol. 36, no. 3, p. 05020005, 2020.

[11] P. Martin, O. Payton, J. Fardoulis, D. Richards, and T. Scott, "The use of unmanned aerial systems for the mapping of legacy uranium mines," Journal of Environmental Radioactivity, vol. 143, pp. 135-140, 2015.

[12] M. Elloumi, R. Dhaou, B. Escrig, H. Idoudi, and L. A. Saidane, "Monitoring road traffic with a uav-based system," in 2018 IEEE Wireless Communications and Networking Conference (WCNC), 2018, pp. 1-6.

[13] C. Graff, "Drone piloting study," University of Italian Switzerland, 2016.

[14] M. Landau and S. van Delden, "A system architecture for handsfree uav drone control using intuitive voice commands," in Proceedings of the Companion of the 2017 ACM/IEEE International Conference on Human-Robot Interaction, 2017, pp. 181-182.

[15] A. R. Fayjie, A. Ramezani, D. Oualid, and D. J. Lee, "Voice enabled smart drone control," in 2017 Ninth International Conference on Ubiquitous and Future Networks (ICUFN). IEEE, 2017, pp. 119-121.

[16] A. U. Bokade and V. R. Ratnaparkhe, "Video surveillance robot control using smartphone and raspberry pi," in 2016 International Conference on Communication and Signal Processing (ICCSP), 2016, pp. 2094-2097.

[17] D. E. Widiyanti and S. Y. Shin, "Holo-drone streaming system," The Korean Institute of Communications and Information Sciences 2021, pp. 1054-1055, 2021.

[18] - "Multi-UAV multi-hololens streaming system," Korean Institute of Communications and Information Sciences 2020, pp. 424-425, 2020.

[19] W. Sun, C. Liu, and J. Zhu, "A remote controlled mobile robot based on wireless transmission," in 2018 2nd IEEE Advanced Information Management,Communicates,Electronic and Automation Control Conference (IMCEC), 2018, pp. 2173-2176.

[20] W. Budiharto, "Design of tracked robot with remote control for surveillance," in Proceedings of the 2014 International Conference on Advanced Mechatronic Systems, 2014, pp. 342-346.

[21] J. Nádvorník and P. Smutnỳ, "Remote control robot using android mobile device," in Proceedings of the 2014 15th International Carpathian Control Conference (ICCC). IEEE, 2014, pp. 373-378.

[22] S. M. Gowda, D. K. Rahul, A. Anand, S. Veena, and V. B. Durdi, "Artificial neural network based automatic speech recognition engine for voice controlled micro air vehicles," in 2019 4th International Conference on Recent Trends on Electronics, Information, Communication Technology (RTEICT), 2019, pp. 121-125.

[23] L. John, N. Vishwakarma, and R. Sharma, "Voice control human assistance robot," in National Conference on Technical Advancements for Social Upliftment, Proceedings of the 2 nd VNC, 2020.

[24] C. Chuixin and J. Xinzheng, "Speech recognize mobile robot," in 2021 5th International Conference on Robotics and Automation Sciences (ICRAS), 2021, pp. 89-93.

[25] Y. Zhou, J. Hou, and Y. Gong, "Research and application of human-computer interaction technology based on voice control

[28] J. Güldenring, P. Gorczak, M. Patchou, C. Arendt, J. Tiemann, and C. Wietfeld, "Skates: Interoperable multi-connectivity communication module for reliable search and rescue robot operation," in 2020 16th International Conference on Wireless and Mobile Computing, Networking and Communications (WiMob), 2020, pp. 7-13. in ground control station of uav," in 2020 IEEE 6th International Conference on Computer and Communications (ICCC). IEEE, 2020, pp. $1257-1262$.

[26] B. Huang, D. Bayazit, D. Ullman, N. Gopalan, and S. Tellex, "Flight, camera, action! using natural language and mixed reality to control a drone," in 2019 International Conference on Robotics and Automation (ICRA), 2019, pp. 6949-6956.

[27] M. Jeong, H. Lee, M. Bae, D.-B. Shin, S.-H. Lim, and K. B. Lee, "Development and application of the smart helmet for disaster and safety," in 2018 International Conference on Information and Communication Technology Convergence (ICTC), 2018, pp. 1084-1089.

[29] B. M. Ravirahul, V. Balaji, K. A. Ram Balaji, S. Gowtham, and S. Surya, "Mind wave controlled assistive robot," in 2021 7th International Conference on Advanced Computing and Communication Systems (ICACCS), vol. 1, 2021, pp. 1507-1509.

[30] J. Kim, M. Lorenz, S. Knopp, and P. Klimant, "Industrial augmented reality: Concepts and user interface designs for augmented reality maintenance worker support systems," in 2020 IEEE International Symposium on Mixed and Augmented Reality Adjunct (ISMAR-Adjunct), 2020, pp. 67-69.

[31] D. Vaquero-Melchor, J. García-Hospital, A. M. Bernardos, J. A. Besada, and J. R. Casar, "Holo-mis: a mixed reality based drone mission definition system," in Proceedings of the 20th International Conference on Human-Computer Interaction with Mobile Devices and Services Adjunct, 2018, pp. 365-370.

[32] D. E. Widiyanti and S. Y. Shin, "Uav-assisted military mapping system using hololens," Korean Institute of Communications and Information Sciences 2020, pp. 1260-1261, 2020.

[33] Z. Cai, M. Chen, and L. Yang, "Multi-source information fusion augmented reality benefited decision-making for unmanned aerial vehicles: A effective way for accurate operation," in 2011 6th IEEE Conference on Industrial Electronics and Applications, 2011, pp. 174178.

[34] S. Sreeram, K. Nisha, and R. Jayakrishnan, "Virtual design review and planning using augmented reality and drones," in 2018 Second International Conference on Intelligent Computing and Control Systems (ICICCS), 2018, pp. 915-918.

[35] M. Unal, E. Bostanci, E. Sertalp, M. S. Guzel, and N. Kanwal, "Geo-location based augmented reality application for cultural heritage using drones," in 2018 2nd International Symposium on Multidisciplinary Studies and Innovative Technologies (ISMSIT), 2018, pp. 1-4.

[36] S. Thon, D. Serena-Allier, C. Salvetat, and F. Lacotte, "Flying a drone in a museum: An augmented-reality cultural serious game in provence," in 2013 Digital Heritage International Congress (DigitalHeritage), vol. 2, 2013, pp. 669-676.

[37] "Rosbridge," Accessed: May. 25, 2021. [Online]. Available: http://wiki.ros.org/rosbridge_suite/

[38] "Unity3d," Accessed: May. 25, 2021. [Online]. Available: https://unity.com/

[39] "MRTK Architecture," Accessed: June. 2, 2021. [Online]. Available: https://docs.microsoft.com/en-us/windows/mixed-reality/ mrtk-unity/architecture/overview?view=mrtkunity-2021-05

[40] J. Jerald, P. Giokaris, D. Woodall, A. Hartbolt, A. Chandak, and S. Kuntz, "Developing virtual reality applications with unity," in 2014 IEEE Virtual Reality (VR), 2014, pp. 1-3.

[41] "Robot operating system - ros," Accessed: May. 25, 2021. [Online]. Available: https://www.ros.org/

[42] M. Quigley, K. Conley, B. Gerkey, J. Faust, T. Foote, J. Leibs, R. Wheeler, A. Y. Ng et al., "Ros: an open-source robot operating system," in ICRA workshop on open source software, vol. 3, no. 3.2. Kobe, Japan, 2009, p. 5. 